\title{
The urethra is a reliable witness: simplifying the diagnosis of stress urinary incontinence
}

\author{
Charles W. Nager
}

Published online: 18 July 2012

(C) The International Urogynecological Association 2012

In 1970, Bates, Whiteside, and Turner-Warwick [1] wrote that: "...the bladder often proves to be an unreliable witness..." and this phrase has been used for more than 40 years as a justification for urodynamic studies (UDS) in the evaluation of urinary incontinence. However, two studies published this year in the New England Journal of Medicine have reported the predictive value of direct observation of fluid loss from the urethra in making the diagnosis of stress urinary incontinence (SUI) [2] and occult SUI [3]. The studies suggest that for SUI or occult SUI, appropriate management decisions can be made with an office evaluation that includes demonstrating urine loss from the urethra without additional UDS.

It probably should not surprise us that SUI is not a condition that requires urodynamic testing. SUI is a urethral problem and current bladder investigations do not investigate urethral conditions very well. Valsalva leak point pressures only indirectly measure urethral pressure, and urethral pressure profilometry typically only measures urethral function at rest and not during SUI conditions. No quantitative or qualitative measure during urodynamics makes the diagnosis of urodynamic stress incontinence (USI); thus, the diagnosis of USI is still dependent on direct visualization or imaging of fluid loss per urethra with cough or Valsalva. The presence of a catheter in the bladder (UDS) adds nothing of value to the diagnosis of SUI, except that it can confirm the absence of a cough or Valsalva provoked detrusor contraction. One could argue, though, that this type of detrusor event would not produce simultaneous fluid loss with cough, because a detrusor smooth muscle is not a striated muscle and cannot contract fast enough to generate

C. W. Nager $(\bowtie)$

UC San Diego Health System,

9350 Campus Point Dr, Ste 2A, Mail Code 0974,

La Jolla, CA 92037-1300, USA

e-mail: cnager@ucsd.edu detrusor pressure at the same time as a cough. Thus, there seems to be a limited role for urodynamics for SUI in the setting of a comprehensive history, office evaluation, and examination. Unfortunately, the current medical model encourages diagnostic studies whether or not they are necessary. This editorial proposes that the diagnosis of uncomplicated SUI and occult SUI by an office stress test and the management of it does not require urodynamic testing in most patients.

\section{The ValUE and OPUS trials}

The Value of Urodynamic Evaluation (ValUE) study was a noninferiority trial involving 11 centers, 53 participating surgeons, and 630 subjects with SUI [2]. Women with uncomplicated demonstrable SUI, a normal post-void residual urine (PVR), and a normal urinalysis were randomized to receive either office evaluation only or office evaluation and urodynamic testing before planned SUI surgery. In the women who received urodynamics, USI was confirmed in $97 \%$. The treatment success rates at 1 year were nearly equivalent in both groups, $77.2 \%$ in the office evaluation only group and $76.9 \%$ in the urodynamic testing group, and the authors concluded that these results argue against routine preoperative urodynamic testing in patients with uncomplicated stress predominant urinary incontinence.

In the Outcomes Following Vaginal Prolapse Repair and Midurethral Sling (OPUS) trial, women without SUI symptoms with stage II-IV prolapse who were planning vaginal prolapse surgery were randomly assigned to undergo vaginal prolapse repair with or without a TVT [3]. All women underwent preoperative standardized fill office-based prolapse reduction stress testing with a fill to $300 \mathrm{ml}$ in the supine position. The catheters were removed and subjects were tested with cough and Valsalva in the supine and then 
standing position with apical prolapse reduction using one or two proctoswabs. Overall, 111 subjects $(33.5 \%)$ had a positive test but in $30 \%(33 / 111)$ of these women it only became positive in the standing position [4].

In the entire group at 3-month follow-up, the prevalence of stress incontinence was significantly lower in the TVT versus sham group (24 and $49 \%$ ). Quite remarkably, women who had a positive preoperative prolapse reduction stress test had a $72 \%$ rate of symptomatic SUI at 3 months compared to a $30 \%$ rate in the sham group (adjusted odds ratio $0.13 ; 95 \%$ confidence interval $0.05-0.34$ ). All subjects had a lower rate of SUI after midurethral sling regardless of preoperative prolapse reduction stress testing, but the results were most pronounced in the positive prolapse reduction stress testing group, and there was modest evidence ( $p=$ 0.06 ) to suggest that patients with a positive prolapse reduction stress test before surgery may have received more benefit than those with a negative test. Although definitive management recommendations were not made by the authors in the OPUS manuscript on whether to do universal, selective, or staged slings at the time of vaginal prolapse surgery in women without symptoms of SUI, it is reasonable to conclude that women with a positive prolapse reduction stress test have a very high rate of postoperative SUI and would likely benefit the most from a midurethral sling.

\section{Performing a standing clinical stress test}

Visualization of fluid loss from the urethra simultaneous with a cough reliably diagnoses SUI. The stress test's likelihood of being positive (sensitivity) is maximized in the standing position with a full bladder and a cough. When patients receive their appointment, ask them to arrive with a comfortably full bladder. After taking the history, have the patient disrobe below the waist and stand near the examining table with their foot on the exam table step or a foot stool to maximize the ability to see the urethra. The examiner needs to bend or squat down and often separate the labia to visualize the urethral meatus. While directly visualizing the urethra have the patient cough vigorously. A Valsalva maneuver can also be performed if the cough test is negative. A positive test is observed loss from the urethral coincident with the cough. Delayed loss is not a positive test but suggestive of possible cough-induced detrusor overactivity. If prolapse is present past the introitus, the test should be performed with reduction of the apex using one or two proctoswabs [3]. If the stress test is positive only with reduction, consider that the patient has a positive reduction testing for occult SUI. This type of standing evaluation also allows observation of maximal prolapse which is recommended in the Pelvic Organ Prolapse Quantification (POPQ) evaluation guidelines [5]. The patient can then complete a measured void in a toilet hat or uroflowmetry toilet. If a bathroom is not connected to the exam room, patients can wrap a full length sheet around their waist to minimize time related to disrobing and redressing for the voiding study. After voiding, the patient returns for the remainder of the evaluation including: a standardized pelvic examination including prolapse exam (POPQ), urethral mobility assessment, and a PVR assessment by catheterization (or ultrasound). By the end of this first visit the physician knows whether the patient has demonstrated SUI or occult SUI, at what volume she was tested (for functional bladder capacity), and her PVR status. A urinalysis can be performed on the catheterized (or voided) specimen. The sum of the voided volume and the PVR is the volume at which the stress test was performed. In our practice, if this volume is at least $200 \mathrm{ml}$ we consider a negative test to be a true negative. If a negative test is obtained at volumes less than $200 \mathrm{ml}$, the option of simple cystometry (inserting a urethral catheter, performing a retrograde fill to maximum capacity, removing the catheter, and performing a standing stress testing) can be considered.

Therefore, in the uncomplicated SUI patient (i.e., without prior surgery or mixed symptomatology) or in the patient with prolapse complaints, all the information available after a single visit should be sufficient to proceed with discussion of management options, including surgical options and even proceeding with surgical scheduling. This "one-stop shopping" approach can be efficient and effective for women with primary SUI or prolapse complaints.

\section{Clinical practice implications}

Arguments against performing a full bladder standing stress test are easy to refute when the alternative for the patient is urodynamic testing to demonstrate stress urine loss. It may be uncomfortable for the provider to bend down or squat down to look at the urethra when the patient is standing, but this should not justify putting the patient through even more discomfort for an invasive urodynamic test with tubes in her bladder and rectum or vagina. This type of comprehensive office evaluation does require a longer first visit to have the patient transfer from the exam room to the toilet and then back to the exam room again, but isn't this preferable to requiring another visit that would be required because she was not full enough to demonstrate the leakage? If patients find the test embarrassing, it is certainly less embarrassing than a urodynamic evaluation. It is our experience that when the purpose of the stress test is explained to the patient, the women readily understand the obvious importance of demonstrating their symptom.

I think many of us realize that financial and economic forces have led to overutilization and overdependence on 
urodynamic testing instead of performing a thoughtful (and less compensated) office evaluation. In the USA, physicians without training and limited understanding of how to perform or interpret urodynamics are encouraged by industry representatives to purchase urodynamic equipment and then request the test when the primary motivation may be to increase practice income. Women are often receiving unnecessary, poorly performed, and uninterpretable investigations with reports that include nonplausible values. Urodynamic testing can provide helpful information about bladder function and is indicated in the setting of diagnostic uncertainty, failed treatment, combined storage and emptying disorders, voiding dysfunction, neurological disease, or uncertainty about the relative contribution of urge and stress components, but its role in straightforward demonstrable SUI is not evident.

In summary, the office-based stress test is a simple noninvasive test that confirms the diagnosis of stress incontinence and occult stress incontinence (with office-based prolapse reduction). It can replace urodynamic testing for SUI in many situations. It should be performed standing, with a full bladder, and with direct visualization of the urethra to maximize its sensitivity and specificity. Based on the evidence, I would argue that the urethra is a reliable witness.

\section{References}

1. Bates CP, Whiteside CG, Turner-Warwick R (1970) Synchronous cine-pressure-flow-cysto-urethrography with special reference to stress and urge incontinence. Br J Urol 42(6):714-723

2. Nager CW, Brubaker L, Litman HJ, Zyczynski HM, Varner RE, Amundsen $C$ et al (2012) A randomized trial of urodynamic testing before stress-incontinence surgery. N Engl J Med 366(21):19871997

3. Wei JT, Nygaard I, Richter HE, Nager CW, Barber MD, Kenton K et al (2012) A midurethral sling to reduce incontinence after vaginal prolapse repair. N Engl J Med 366(25):2358-2367

4. Nager C (2011) The value of the preoperative prolapse reduction stress test in women without stress incontinence symptoms undergoing vaginal prolapse surgery with or without a TVT: results from the OPUS trial. Female Pelvic Med Reconstr Surg 17(5 Suppl 2): S53

5. Bump RC, Mattiasson A, Bø K, Brubaker LP, DeLancey JO, Klarskov $P$ et al (1996) The standardization of terminology of female pelvic organ prolapse and pelvic floor dysfunction. Am J Obstet Gynecol 175 (1): $10-17$ 